\title{
Modified Index Method in Scenarios of Regional Socio-Economic Development
}

\author{
Panasyuk M.V. \\ Pudovik E.M. \\ Malganova I.G. \\ Kazan (Volga Region) Federal University
}

\section{Doi:10.5901/mjss.2014.v5n18p331}

\section{Abstract}

\begin{abstract}
The paper proposes research approach to analysis of regional socioeconomic system development. The system of estimated parameters of regional socioeconomic system state in the context of municipalities and local government entities is substantiated. It allows determining the optimal variant of regional system development and the level of municipalities' economic development based on medium term (5 - 10 years) scenarios. The authors have elaborated modified index method for forecasting the regional socio - economic system state based on analysis of a degree of change of socio-economic development parameters, depending on the scenario option. The paper shows the mechanism of the proposed method usage and the results of the individual expert judgments colligation. The prospects of raising the level of socio - economic development of the Republic of Tatarstan municipalities are determined. By prioritizing, basic (needed for implementation of any scenario) and special (requiring urgent actions for each of the scenarios) directions of region development are offered. The directions of development are considered from the standpoint of the four types of territorial differentiation of socio-economic development of the region ("North-South", "East-West", "center-periphery", and "mono - multiethnic regions"). Major trends of probable variations in intraregional disparities in socio-economic development of the Republic of Tatarstan are related to strengthening the role of semi-periphery in the regional development and reduction of differentiation of socio-economic development level between the central and peripheral zones, as well as to the ongoing regional spatial polarization of the Republic.
\end{abstract}

Keywords: regional economics, socioeconomic development, index method, regional forecast

\section{Introduction}

Region with its own unique character and level of socio-economic development is more vulnerable, but very adaptable spatial system, especially in conditions of alternative (economic, social, political and other) transformations of modern society $[1,7$, and 8$]$. That is why there is a need to accomplish a science-based assessment of the effectiveness of these transformations on the regional and interregional levels.

Research of state of socio-economic system and dynamics of its development is important for territories of any hierarchical level. In current conditions we need to study the whole diversity of processes taking place with the active participation of people within the region, municipal and local formations $[2,4$, and 6$]$. Tracking these processes, you can identify the main principles of functioning and development of intra-regional territorial units, originality and, to some extent, the uniqueness of each of them, territorial differentiation of population life quality. So, the constructive slogan «unity in diversity» becomes very actual. It is more useful to learn how to use the complementarity of local differences, peculiarities and regionalisms in forming organic, grass-roots and because of those strong relationships between regions and locations, than to erase, to suppress or to unify these differences.

Note that in conditions of permanent socio-economic changes the possibility of multiple further social development increases greatly. In this regard scenario forecasting of trends in socio-economic system of region from the point of its territorial differentiation is very important, and a complex of predictive scenarios describing different ways of development depending on value of key factors forms the holistic forecast.

Comprehensive predictive study of socioeconomic development of the region (on the example of territorial differentiation of social-economic system of the Republic of Tatarstan) is based on comparative assessment and analysis of the proposed forecast scenarios for the medium term (up to 2020). Classification of the municipalities of the Tatarstan, based on collected data of these territorial units and its mapping are formed on the basis of resulting indicator of forecasted index of regional socioeconomic development. 
A study of the development of territorial socioeconomic system of the region requires the use adapted instrumental and methodological approaches to the specificities of the polyethnic region, which focused on monitoring and forecasting of intra-regional socioeconomic and territorial division.

\section{Methodology}

The analysis of methodical and methodological approaches to monitoring and forecasting regional socioeconomic systems of a region and its territorial differentiation $[3,9$, and 10] showed that the most acceptable and adequate is the combination of methods of logic simulation (including construction of the forecast scenarios), mathematical-statistical [5] and expert estimates.

The paper proposes modified mathematical-statistical index method for monitoring and forecasting territorial socioeconomic disparities in the region taking into account the proposed regional characteristics the rate of change of indicators depending on the scenario options. Our research has led to conclusions on the need to develop a method of expert estimates based on the questionnaire, adapted for the purposes of regional forecast.

Modified mathematical-statistical index method of forecasting of territorial differentiation in life quality of regional population includes the following sequence of stages:

The 1st stage. Preliminary study (selection of the prior components and preparation of indicators, retrospective analysis of regional socio-economic development).

The 2 nd stage. Formation of the system of forecast hypotheses

The 3rd stage. Formation of the prognostic models' system of regional development

The 4th stage. Analysis of forecast scenarios

It should be noted that the system of «significant» key events in the medium term, obtained using the method of expert estimations appears to be the basis for the formation scenarios of territorial socio-economic development of the region.

For correct construction of the forecast scenario variants it is offered to include the special stage of investigation the formation of system of forecast hypotheses. Its goal is the definition of «significant» event from the standpoint of socio-economic development of the Republic of Tatarstan events for the medium term (2016-2020). The analysis of the state forecasting documents that determine the prospects of socio-economic development of the Republic of Tatarstan, and also the expert selection of key events helped to make the following conclusions: in the next five years, in Tatarstan it is possible to assume the following events that define the trajectory of the development of territorial social-economic systems:

I. Reorientation of social policy from the growth of social security in the direction of the growth of productive employment;

II. Strengthening of investment, information and innovative orientation of economic development after the Universiade-2013 in Kazan;

III. «Greening» of public relations (Tab.1).

Table 1. Structure of territorial development scenarios of the Tatarstan Republic

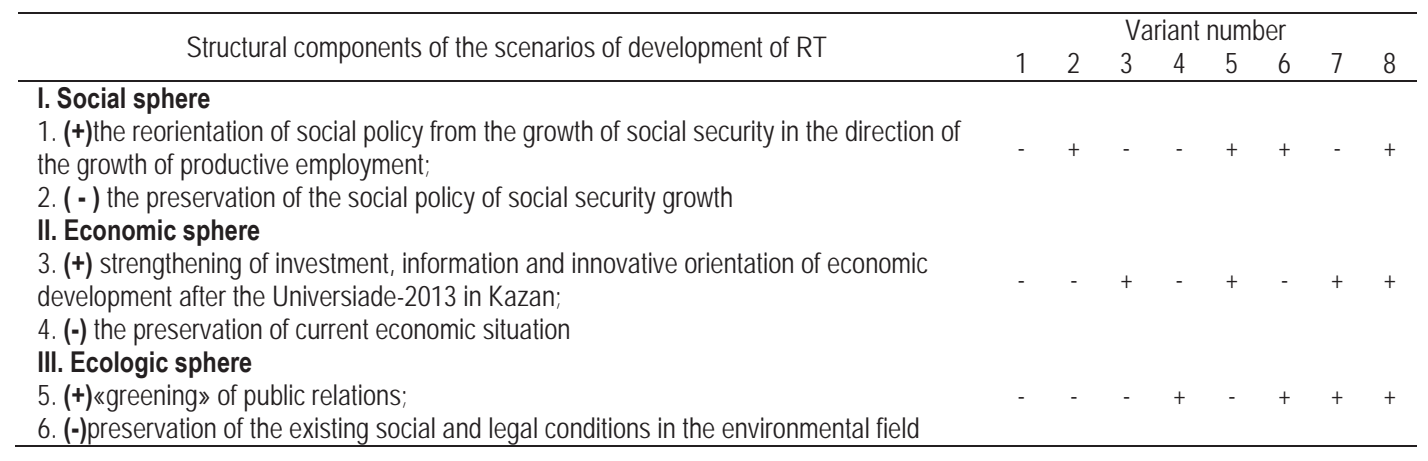

In medium term the highlighted events will have a strong influence, including case of interacting with each other. Every possible variant of scenario development is a complex compound. Its structure will be determined by happen $(+)$ or does 
not happen (-) each of the selected event (Tab. 1). Eventually eight different scenarios of development were formed, and the implications of their implementation should be assessed. In turn, obtained scenarios of development of the Republic of Tatarstan were grouped in five proposed scenarios of the region development: inertial, stabilization, moderately optimistic, optimistic and program one.

The authors proposed the system of impact characteristics of each of the presented development scenarios of the Republic of Tatarstan on the socio-economic system of the region in areas: «Direction», «Power», «Delay», «Duration», «Cycling of events», «Regional weights» and «Random component (risks)».

The «Direction» is considered to be positive if the occurred event leads to improvement; the «Power» refers to the change of the indicator under the influence of events at the time of maximal effect; the "Delay» - estimate of the length of the initial time interval at which the effect may be considered insignificant; the «Duration» estimates of the length of the time interval on which the impact can be considered significant; the «Cycling of events» - assessment of the impact of periodic fluctuations of events affecting the socio-economic development of the Republic; the «Regional weight» assessment of the influence of regional characteristics (specificity) on territorial social-economic system of RT; the «Random component» - assessment of the influence of random factors.

The generalized characteristic «the degree of indicators' changes» is the resultant and reflects the importance of the impact of the analyzed variants of events on the level and speed of socio-economic development. For the calculation we can suggest the following general formula (1):

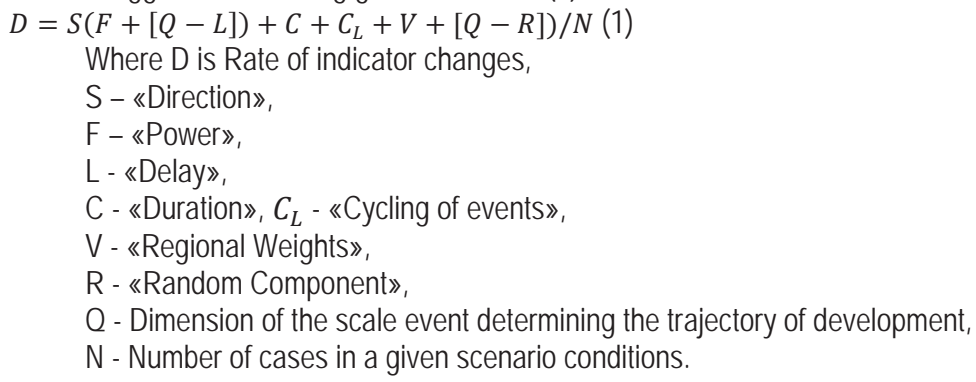

\section{Conclusions}

Development of characteristics' system, which indicates changing of socio-economic development of the region's population under the impact of the proposed forecasting scenarios helps to identify most appropriate moderatelyoptimistic scenario.

This scenario assumes occurrence and development in medium terms the following events: increased investment, information and innovative orientation of economic development, «Ecologization» of legal relations, while maintaining a policy of growth of social security. The likelihood of a moderately optimistic scenario is defined according to a survey by expert group (seven experts identified this scenario as the most likely; two suggested that the most likely is the stabilization scenario; one expert estimated the inertial scenario as most real one).

Classification of municipalities and economic regions of the Republic of Tatarstan according to the forecast indices of socio-economic development revealed a significant territorial differentiation.

Territorial stratification integral forecast of quality of life index show unfavorable situation in South-Eastern economic region (extremely low index of socio-economic development); in the best position are Privolgskiy and Zakamski regions (the average index of socio-economic development) and Northeast (moderately average index of socio-economic development) region. Socio-economic leaders at intra-regional level are the North-Western and Predkamski economic regions (high index of socio-economic development).

This distribution is explained by scenario-based development of ecological and economic sphere of the region, as well as the inertia of the social component of regional development. The number of municipalities with the smallest value of the forecasting index compared with the actual values of the 2005 index remains unchanged, however, the group of municipalities with a maximum value will undergo changes; the leader is unchangeable - Kazan.

The investigation identifies main directions of improving life quality of population of the municipalities and economic regions of the Republic of Tatarstan. In preference manner the following main (necessary in the implementation of any of the scenarios), and special (urgent measures for each of the scenarios) directions of development of the region are marked out. The directions of development are considered from positions of four types of territorial differentiation of social-economic development of Tatarstan («North-South», «West-East», «Center-Periphery», and «Mono - Poly-ethnic 
Regions»). Each direction involves a set of measures aimed at improving the system of indicators of socio-economic development at intra-regional level.

Major trends of probable variations in intraregional disparities in socio-economic development of the Republic of Tatarstan are related to strengthening the role of the semi-periphery in the regional development and reduction of differentiation of socio-economic development level between the central and peripheral zones, as well as to the ongoing regional spatial polarization of the Republic.

\section{References}

Bartlett W., Cipusheva Hr., Nikolov M. and Shukarov M. (2010). The quality of life and regional development in FYR Macedonia. Croatian Economic Survey, 12 (1), 121-162.

Charles V., Zegarra L.F. (2014) Measuring regional competitiveness through Data Envelopment Analysis: A Peruvian case. Expert Systems with Applications, 41(11), 371-5381

Hongshan, Ai J.S. (2013) Research on regional economy prediction based on partial least squares support vector regression. International Journal of Applied Environmental Sciences, 8 (13), 1645-1652

Huggins R. , Izushi H., Prokop D., Thompson P. Regional evolution and waves of growth: A knowledge-based perspective. Expert Systems with Applications , 41 (12), 5573-5586

Li, Z., Wang, P. (2013). Comprehensive Evaluation of the Objective Quality of Life of Chinese Residents: 2006 to 2009. Social Indicators Research, 113 (3), 1075-1090.

Panasyuk M.V., Rudenko A.V. (2008). Analysis of "center-periphery" system development (evidence from the Republic of Tatarstan). Bulletin of the Russian Academy of Sciences. Geography, 1, 60-72.

Parr J.B. (2012). Spatial-structure differences between urban and regional systems. Annals of Regional Science, 49(2), 293-303.

Safiullin, L.N., Bagautdinova, N.G., Safiullin, N.Z., Ismagilova, G.N. (2012) The Development of welfare theory in conditions of changes in the quality of goods and services. World Applied Sciences Journal, 18, 144-149.

Santana N.B, Aparecida Do Nascimento Rebelatto D., Périco, A.E., Mariano, E.B. . (2014) Sustainable development in the BRICS countries: An efficiency analysis by data envelopment. International Journal of Sustainable Development and World Ecology, 21(3), 259-272

Vlasyuk L.I., Minakir P.A. (2013) Regional long-term forecasts: A synthesis of technological and economic approaches. Studies on Russian Economic Development, 24 (2), 99-107 